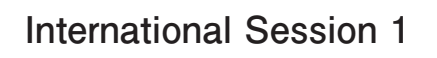

\title{
CLARITHROMYCIN INHIBITS TNF- $\alpha$-INDUCED MUC5AC MUCIN GENE EXPRESSION VIA MKP-1
}

\author{
Said Ahmad Shah, Hajime Ishinaga, Kazuhiko Takeuchi \\ Department of Otorhinolaryngology-Head and Neck Surgery, Mie University Graduate School of Medicine
}

Introduction: Clarithromycin (CAM) is a 14-membered macrolide antibiotic. Low dose, long term macrolide therapy is effective in patients with chronic airway diseases, such as diffuse pan-bronchitis, chronic bronchitis, chronic asthma and chronic sinusitis. However, the mechanism underlying the clinical efficacy remains unclear. The purposes of this study are, to investigate the mechanisms underlying the effects of macrolides on mucin gene expression induced by tumor necrosis factor- $\alpha$ (TNF- $\alpha$ ).

Method: Human bronchial epithelial cells lines NCI-H292 were treated with CAM $25 \mu \mathrm{g} / \mathrm{ml}$ for $18 \sim 24$ hours or with co-treatment of TNF- $\alpha$. Real time quantitative polymerase chain reaction $(\mathrm{PCR})$ was performed to detect the relative quantity of MUC5AC and MKP-1 (mitogen activated protein kinase phosphatase-1) mRNAs levels. MKP-1 specific siRNA was used to confirm whether upregulated MKP-1 is involved in MUC5AC gene expression induced by TNF- $\alpha$. Total and phosphorylated MAPKs (p38) were assessed by western blot analysis of cell lysates.

Results: CAM treatment resulted in the induction of MKP-1 mRNA. In addition, CAM downregulated p38 phosphorylation. However, knockdown of MKP-1 enhanced p38 phosphorylation.

Conclusion: The results of present study demonstrated that CAM induces MKP-1 which downregulates p38, and in turn leads to suppress TNF- $\alpha$-induced MUC5AC transcription. This study provides new insights into the important role of MKP-1 induced by CAM in regulating mucin gene expression. 